\title{
Les dangers de la navigation et de la pêche dans l'Atlantique Français au $18^{\mathrm{e}}$ siècle
}

\section{Nicolas Landry}

\begin{abstract}
A central theme in the historiography of the Ancien Régime in Canada has always been the ocean crossing between France and New France. Despite the advancement of scientific knowledge during the $18^{\text {th }}$ century, navigation remained a major challenge for those wishing to travel from France to its overseas colonies. Storms were a constant threat, as was piracy and, for much of the era, war. Marine disasters were frequent and took a heavy toll among the officers, crews and passengers. More comprehensive research on shipwrecks during the French Régime in Canada is needed. The present article seeks to further our knowledge of the circumstances prevailing aboard endangered ships, how local authorities responded to the disasters on their shores, especially care for survivors and salvage of cargoes, and how they reported these events and challenges to Versailles.
\end{abstract}

Durant le $18^{\text {e }}$ siècle, par la nature même de leurs lieux géographiques, les colonies françaises du Golfe Saint-Laurent dépendent grandement de la métropole pour leur approvisionnement, leur commerce et leur défense. Les traversées de l'Atlantique sont donc nombreuses, avec tous les dangers que cela implique. Laurent Huet mentionne entre autres que « les pertes matérielles suite à la déroute d'un bâtiment au large des côtes de Nouvelle-France sont fortement ressenties des deux côtés de l'Atlantique, pertes de vivres, de matériel, de personnes, etc. ${ }^{1}$ " Quant à Gilles Proulx, il estime que " la liste des incidents pourrait s'allonger indéfiniment ${ }^{2}$. » Kenneth Banks, lui, estime qu'on en connaît peu sur les routes maritimes et les conditions de navigation rencontrées par les milliers de matelots, traversant l'Atlantique au $18^{\mathrm{e}}$ siècle $^{3}$.

1 Laurent Huet, «L'économie du naufrage en Nouvelle-France entre 1715 et 1763 », Mémoire de Master I, Université de Poitiers, Promotion 2012, p. 16. Pour les naufrages de navires de pêche à Terre-Neuve, voir Laurier Turgeon, " Naufrages de terre-neuviers bayonnais et luziens (1689-1759) », Bulletin de la Société des Sciences, des Lettres et des Arts de Bayonne, nouvelle série no 134 (1978), pp. 115-123.

2 Gilles Proulx, Entre France et Nouvelle-France, La Prairie, Éditions Marcel Broquet, 1984, p. 73.

3 Kenneth J. Banks, Chasing Empire Across the Sea. Communications and the State in the

The Northern Mariner/Le marin du nord XXV, No. 1 (January 2015), 43-64 
Dans la très grande majorité des échanges de correspondance traversant l'Atlantique dans un sens comme dans l'autre, combien de fois ne mentionne-t-on pas des incidents maritimes, les contraintes que les traversées imposent, etc. ${ }^{4}$. Bien que nous ne tentions pas de définir un territoire d'étude aux contours fixes entre la France et la Nouvelle-France, trois entités géographiques retiennent plus particulièrement notre attention soit l'île Royale, le golfe et le fleuve Saint-Laurent de même que Saint-Pierre et Miquelon.

Ce texte n'aspire pas non plus à l'exhaustivité en ce qui a trait à un décompte combien souhaitable des pertes de navires à l'époque du Régime français. Nous optons plutôt pour trois démarches distinctes mais reliées entre elles. D'abord, nous proposons une relecture d'une vingtaine de documents d'archives relatant quelques incidents maritimes significatifs. L'objectif fondamental de cette première démarche consiste à recréer ces moments d'effroi que vivent les occupants et les scénarios à envisager pour éviter le pire. Ils démontrent aussi l'impact qu'ont ces événements sur les populations côtières et sur les maigres ressources des administrations coloniales de l'époque. La deuxième démarche propose une brève analyse quantitative d'un petit nombre incidents maritimes et des pertes subies en temps de guerre ${ }^{5}$. Précisons cependant que les sources utilisées à cette fin ne constituent qu'une parcelle de celles existantes dans les centres d'archives départementales de France tels La Rochelle, Nantes, Rennes, Bayonne ou encore Bordeaux et Marseille. Le portrait que nous traçons ne peut donc être que partiel.

Finalement, sous forme d'annexe, nous abordons certaines réglementations visant à rendre la navigation plus sécuritaire dans la grande pêche à Terre-Neuve. Il s'agit ici d'une problématique véritablement atlantique, puisque ces incidents et ces règlements peuvent se produire ou s'appliquer à l'ensemble du territoire que constitue alors l'Atlantique Français.

\section{Quelques descriptions d'incidents maritimes}

Avant d'aborder la question des incidents maritimes, l'on est en droit de se questionner sur l'évolution des connaissances hydrographiques des navigateurs français du $18^{\mathrm{e}}$ siècle. L'historien James Pritchard a déjà publié un article élaboré sur cette question, en cernant habilement les défis empêchant les nouvelles connaissances de

French Atlantic, 1713-1763, Montréal et Kingston, McGill-Queen's University Press, 2002, p. 66.

4 Certains travaux en archéologie sous-marine ont eu pour résultats d'accroître nos connaissances sur les épaves repérées suite à des naufrages du $18^{\mathrm{e}}$ siècle. Par exemple, l'excellente thèse doctorale de Charles Dagneau, "La culture matérielle des épaves françaises en Atlantique nord et l'économie-monde capitaliste, 1700-1760 », thèse de doctorat en Anthropologie, Université de Montréal, 2008. Son étude porte sur quatre épaves françaises datant de la période 1704-1760.

5 À noter que les données ayant servies à cette analyse proviennent du site web http://naviresnouvellefrance.net/. Ce site est l'œuvre de Charles Vianney Campeau. Bien que ses données débutent au moment de la fondation de la Nouvelle-France, nous nous limitons à celles du $18^{\mathrm{e}}$ siècle. 
trouver une oreille attentive auprès du monde maritime de l'époque ${ }^{6}$. Colbert, à titre de ministre de la Marine, aspirait à former un groupe de pilotes et de capitaines pour les voyages aux colonies. Banks rappelle qu'en 1685-86, Jean Deshayes entreprend « an extensive triangulation Survey » du Saint-Laurent et de la Péninsule de Gaspé. Sa carte est toujours en usage en $1716^{7}$.

Avec les années, l'on note la conception de nombreuses cartes, on aspire à réaliser l'hydrographie des eaux menant en Nouvelle-France, etc. Qui ne connaît pas les nombreuses cartes de Jean-Baptiste-Louis Franquelin et les tentatives d'enseignement de l'hydrographie à Québec par Martin Boutet? Les succès et les outils disponibles demeurent néanmoins limités. Encore en 1720, le dépôt de la Marine ne compte aucune carte des eaux environnantes de l'île Royale. Toutefois, durant les années $1730^{8}$, des efforts pour cartographier le golfe et le fleuve Saint-Laurent se déroulent sous la responsabilité de Richard Testu de La Richardière.

Mais l'arrivée du marquis de La Galissonière, à titre de directeur du service hydrographique de la Marine après 1748, donne lieu à une série de publications de cartes et d'ouvrages hydrographiques. On pense entre autres à l'œuvre de Joseph-Nicolas Bellin'. Pertinents à notre texte, citons la publication Le pilote Américain Septentrional par George-Louis Le Rouge en 1778 et l'expédition de Liberge de Granchain à TerreNeuve en 1784. Les Britanniques, eux, ne nomment un hydrographe pour la Navy qu'en 1795. Le grand défi consistant à déterminer la longitude de manière adéquate en mer, n'est résolu que durant le dernier quart du $18^{\mathrm{e}}$ siècle $^{10}$.

\section{En pleine mer!}

Les dommages découlant des incidents maritimes du $18^{\mathrm{e}}$ siècle sont multiples et nécessitent de nombreuses manœuvres des équipages; « voiles déchirées, mâts cassés » exigent l'usage des « pompes pour évacuer l'eau, boucher les fissures avec de l'étoupe, colmater la voie d'eau avec une plaque de plomb», etc. Les conséquences sur les cargaisons sont toutes aussi nombreuses; " perte de sel, de vivres, des marchandises gâtées par l'humidité et les infiltrations d'eau ${ }^{11}$. » Il est également important de noter qu'à compter de 1713, les administrateurs des ports de France tentent de faire partir les

6 James Pritchard, « Le problème de l'Amérique du Nord dans la science nautique française aux XVII ${ }^{\mathrm{e}}$ et XVIII ${ }^{\mathrm{e}}$ siècles ", dans Martine Acerra, José Merino, Jean Mayer (eds.), Les marines de guerre européennes XVII ${ }^{e}-X V I I I^{e}$ siècles, Paris, Presses de l'Université de ParisSorbonne, 1985, pp. 345-358.

7 Banks, Chasing Empire, p. 72.

8 Olaf Uwe Janzen, «Of Consequence to the Service: The Rationale Behind Cartographic Surveys in Early Eighteenth-Century Newfoundland », The Northern Mariner / Le marin du nord, XI, n 1 , January 2001, p. 2.

9 Pritchard, «Le problème de l'Amérique du Nord », pp. 334, 347, 351-353

10 Janzen, «Of Consequence to the Service », p. 2. Cet auteur est d'avis qu'en dépit de l'existence de cartes, les pilotes et capitaines continuent de donner la priorité à l'expérience de navigation pour connaître les vents dominants, les courants océaniques, etc.

11 Proulx, Entre France et Nouvelle-France, p. 70. 
navires en mai pour leurs voyages au Canada. On souhaite bien sûr qu'ils repartent de Québec, avant que ne se forment les glaces sur le Saint-Laurent ${ }^{12}$.

Aux deux extrémités de la guerre d'Espagne, deux épisodes de naufrages ont laissé passablement de détails dans les archives autant pour la Ville-Marie que pour le Comte de Vermandois. La Ville-Marie, de La Rochelle, jauge entre 200 et 300 tonneaux lors de son trajet entre La Rochelle et Québec. Le récit de son naufrage est l'œuvre de deux marchands de La Rochelle, passagers du navire, soit Antoine Chalumeau et Jacques Le Clerc ${ }^{13}$. En résumé, après le départ de Québec, le mauvais temps finit par provoquer une voie d'eau devenue apparente " une fois dépassé le Grand banc de Terre-Neuve ». Afin de se maintenir à flot, il est alors convenu de « jeter quantité de marchandises à la mer ». Cette sage décision permet au navire d'atteindre le port de Kinsale en Irlande, où le bâtiment doit radouber avant de repartir pour La Rochelle. Mais une fois le long des côtes de Bretagne, la Ville-Marie " s'affala sur des rochers ». Heureusement, une trentaine de passagers, dont vingt-et-un hommes d'équipage s'en tirent indemnes. De là, le navire endommagé aborde à " liste des Saints » (île de Sein?) en face d'Audierne. Le curé sur l'île de Sein, J. Garnier, délègue prestement six bateaux de pêche, dans l'espoir de rescaper les survivants et on rapporte que six ou sept hommes « furent trouvés sur les rochers ». Étant confronté au fait que les trois quarts du navire sont à la dérive, il n'est possible de récupérer que « quelques pièces de peaux blanches et de martres de peu de valeur et une cassette de papiers ${ }^{14}$. $\gg$ Au final, entre treize et seize hommes périssent dans ce naufrage dont le capitaine et le maître ${ }^{15}$.

En ce qui a trait au Comte de Vermandois, également de La Rochelle, il jauge entre 300 et 400 tonneaux et navigue sous le commandement du capitaine Pierre Bousselot, à l'emploi des propriétaires Bonfils Frères ${ }^{16}$. La destination initiale est alors Québec mais un coup de vent oblige le navire à « se mettre à la cape ${ }^{17}$ » et un deuxième fracture la jambe du pilote Étienne Vallet et emporte le matelot Jacques Doussou. Dans l'espoir de rester hors de l'eau, l'équipage lance par-dessus bord " plusieurs pièces d'artillerie et éléments du gréement dont quatorze pièces de canons, certains avec leurs

12 Banks, Chasing Empire, pp. 57-58.

13 Sur cette famille de négociants de La Rochelle, voir Brice Martinetti, Les négociants de La Rochelle au XVIII siècle, Rennes, Presses universitaires de Rennes, 2013, pp. 80, 156 et 281.

14 La famille Pacaud occupe une grande place dans le négoce de La Rochelle au XVIII ${ }^{\mathrm{e}}$ siècle, voir Martinetti, Les négociants de La Rochelle, p. 101 et autres.

15 Site web de Charles Vianney Campeau, Navire $n^{\circ} 1280$, La Ville-Marie. Sur la question des mortalités en mer, un colloque doit retenir notre attention soit celui intitulé Des bateaux et des morts, tenu à l'Université du littoral Côte d'Opale au printemps 2015 (13 mars et 22 mai). Soulignons entre autres les communications de Jacques Peret, « Les rivages de la mort à travers les naufrages entre Loire et Gironde (XVII ${ }^{\mathrm{e}}$-XVIII ${ }^{\mathrm{e}}$ siècle) » et d'Agathe Leyssens, « Reconnaître la mort en mer (XVIIIe siècle, Côte d'Opale) ».

16 Sur cette famille de négociants de La Rochelle, voir Martinetti, Les négociants de La Rochelle, p. 103 et autres.

17 Se mettre à la cape : situation d'un bâtiment qui a la barre du gouvernail sous le vent, et qui ne conserve que très peu de voiles, afin de présenter le côté et de ne plus faire de route. 
affûts, des balles, de la poudre, vingt-huit palans, quatorze pinces, et même le canot avec ses avirons etc. ». Pour espérer maintenir les mâts, l'équipage se résout à couper un grelin et plusieurs palans. Mais les grands vents se maintiennent, obligeant l'équipage à garder «les deux voiles basses ». Sans doute au grand désespoir du capitaine, on réalise que «l'on pompe de l'eau couleur de vin » puisque l'eau de mer a déjà envahi "la grande chambre, le pont et l'entre deux ponts ». Le capitaine, craignant que « les marchandises en soient gâtées ", dresse dès lors un procès-verbal de la cargaison et des événements « en présence des officiers, de l'équipage et des passagers ». Malgré tout, le navire atteint Plaisance à Terre-Neuve, d'où il ne repart que pour affronter plusieurs coups de mer qui « démâtent le navire du mât de hure avant et deux mâts de perroquet ». C'est ici que s'arrête cette description dans le rapport ${ }^{18}$.

\section{Des tentatives de réglementation de la navigation en métropole}

En France même, les années 1720 semblent susciter de vives inquiétudes en termes de réglementation pour encadrer la gestion des naufrages et des navires échoués. En 1723, une lettre du futur Intendant de Nouvelle-France, Gilles Hocquart, souligne la fréquence des naufrages près des côtes de Barfleur et de La Hougue. Il se réfère entre autres à la perte d'un terre-neuvier et déplore le manque de connaissances des maîtres et des pilotes ${ }^{19}$. Ce qui nous ramène aux observations de Pritchard en introduction de cette section.

Également, dès le mois suivant, sont proclamées les condamnations des habitants de Saint-Vigar (France) et de leurs seigneurs riverains au sujet de marchandises échouées sur le rivage. Il est donc ordonné à tous les seigneurs et habitants des paroisses longeant la mer et la rivière de la Seine, de nommer une ou deux personnes pour surveiller les « échouements et naufrages » pouvant survenir le long de leurs côtes. Les personnes en question doivent ensuite rendre ces événements publics et en dresser un procès-verbal ${ }^{20}$. D'ailleurs, en ce même mois de juin 1725 , un procès est intenté à des habitants des Sables-d'Olonne pour pillage d'effets naufragés ${ }^{21}$.

18 Site web de Charles Vianney Campeau, Navire non ${ }^{\circ}$ 1423, Le Comte de Vermandois.

1931 mai 1723, Lettre de Hocquart au ministre, Bibliothèque et Archives Canada (BAC) BACMG2-B3, bobine C11618, vol. 286, f 166-167v.

2018 juin 1723, Condamnations des habitants de Saint-Vigar, BAC-MG6-A9, bobine F1709, vol. 120 , f $32-32 \mathrm{v}$.

2120 juin 1725, Lettre de Jean-Baptiste Des Gallois de La Tour, Intendant de Poitiers, à Maurepas, BAC-MG2-B3, bobine C11619, vol. 307, f 232-233v. Dans son étude, Jacques Péret exploite un corpus de 750 dossiers de procédures de naufrages survenus entre 1680 et 1789 sur " les rivages des deux amirautés de La Rochelle et de Marennes ». Environ cinquante-six pourcent de ces navires sont de "petites unités jaugeant moins de cinquante tonneaux, alors que onze pourcent sont des gros trois-mâts jaugeant plus de 200 tonneaux. Dans plus de $80 \%$ des naufrages, les mauvaises conditions météorologiques sont en cause ». Jacques Péret, "Les étrangers naufragés sur le littoral charentais (XVII ${ }^{\mathrm{e}}-\mathrm{XVIII}{ }^{\mathrm{e}}$ siècles) », Annales de Bretagne et des Pays de l'Ouest, 117-1, 2010, pp. 1-10. Pour sa part, Sylvain Coindet utilise les procès-verbaux de trente-trois naufrages survenus entre 1721 et 1791 dans son étude sur l'île de Sein. Il est plutôt d'avis que « le naufrage n'est pas la conséquence 
De plus, sans doute dans l'espoir d'amoindrir les risques d'incendie à bord des navires, une ordonnance royale de 1737 défend aux membres d'équipages des navires de pêche « d'embarquer des paillasses, ni autres meubles où il y ait de la paille, foin ou autres herbes sèches ${ }^{22}$. » Deux années plus tard, en 1739, d'autres ordonnances et arrêts sont publiés, touchant les « gens de mer et passagers morts en mer ». On parle ici d'un règlement ordonnant la recherche des soldes et produits d'inventaires des gens de mer décédant « tester » (testament), durant leurs voyages sur les bâtiments marchands. Les articles en question peuvent sans doute inclure des hardes et autres effets. Finalement, on réitère le fait que les " produits des bris et naufrages » reviennent aux invalides de la Marine $^{23}$.

\section{En remontant le fleuve vers le Canada!}

La route maritime France-Canada se divise en deux étapes; la première se situe entre les ports de France et les Grands Bancs de Terre-Neuve soit environ $3400 \mathrm{~km}$. C'est ce qu'on peut qualifier de segment européen du trajet. Avant d'aborder les descriptions de quelques naufrages se produisant sur la route du Canada, quelques précisions territoriales s'imposent. Nous empruntons nos paramètres de l'étude de La france $^{24}$ qui subdivise son laboratoire de recherche en quatre sections que sont MontréalQuébec, Québec-Tadoussac, Tadoussac-Sept-Îles et Sept-Îles-île d'Anticosti. Toutefois, nous excluons le segment Montréal-Québec de notre étude. Également, au lieu de refaire un exercice quantitatif portant sur le nombre de naufrages survenus dans l'axe Québec-Île d'Anticosti, nous référons plutôt le lecteur aux résultats de recherche de Lafrance. À noter que certains de ces naufrages impliquent des navires mentionnés dans notre article et nous le signalons en note à l'occasion. Lafrance ne fait pas de distinction entre les incidents maritimes survenus en temps de guerre ou de paix. En tout et partout, il identifie environ trente-cinq lieux d'épaves entre Montréal et l'entrée du Golfe SaintLaurent pour la période 1650-1760.

Notre tour d'horizon de ces quelques incidents maritimes sur le Saint-Laurent débute en 1679, alors qu'un vaisseau de France apportant des provisions et du matériel médical destiné à l'hôpital de Montréal fait naufrage. Pour sa part, l'année suivante, la

directe de la tempête » mais plutôt de " l'accumulation de plusieurs facteurs qui, en se combinant, conduisent à la perte du navire et, dans un certain nombre de cas, à la mort de l'équipage ». Sylvain Coindet, «Les naufrages sur l'île de Sein au XVIII ${ }^{\mathrm{e}}$ siècle : une lente évolution vers le sauvetage », Annales de Bretagne et des Pays de l'Ouest, 113-1, 2006, pp. 88, 90-91.

22 Lucien Marie, "Mourir à Terre-Neuve », Troisièmes Journées d'Histoire de la Grande Pêche, (Granville, 18-19 mars 2005), Saint-Lô. Société d'archéologie et d'Histoire de la Manche, 2007, p. 200.

2328 août 1739, Arrêts, édits et ordonnances de la Marine à Vincennes. BAC-MG1-Série 3S2, vol. 48, dossier 165 bis. 8 pages. Voir aussi 23 août 1739, Versailles. BAC-MG6-A2, bobine C9183, vol. 270, pp. 148-159.

24 Jean Lafrance, Les épaves du Saint-Laurent (1650-1760), Montréal, les Éditons de l'Homme, 1972. 
perte du Saint-Joseph, prive les « sieurs intéressés dans la ferme » du roi $^{25}$ de certaines provisions. Quelques années plus tard, une tempête emporte le Corossol vers un destin fatal près de l'actuelle ville de Sept-Îles. Ce navire avait pourtant respecté la consigne de naviguer en convoi entre Québec et la France ${ }^{26}$. L'on s'en doute, découper les naufrages selon des territoires précis demeure un défi que nous ne prétendons par relever ici avec brio. Par exemple, où classer le cas du navire les Quatre Frères? En 1723, revenant alors de France, ce navire, appartenant à la Compagnie de l'île Saint-Jean, fait naufrage vers le 11 novembre près de l'île Miscou. Cela s'explique par le fait que cette île fait partie de la concession exploitée à partir de l'île Saint-Jean ${ }^{27}$.

Toujours au Canada, en 1729, le naufrage de l'Éléphant permet au sieur Debois de se signaler favorablement auprès des autorités car il « s'est prêté avec toute la vivacité et l'attention possibles au déchargement des effets naufragés, à leur arrangement dans les magasins et qu'il a contribué efficacement à empêcher que rien ne soit pillé, ni égaré de tout ce qui avait été sauvée ${ }^{28}$. Selon Banks, le capitaine de port de Québec, Richard Testu de La Richardière, effectue alors trois voyages de sauvetage pour récupérer ce qu'il peut, incluant des canons et des munitions. On le recommande même pour recevoir une gratification supplémentaire ${ }^{29}$. Ce qu'il faut retenir de cet épisode est le fait qu'aux yeux des riverains de Nouvelle-France, un naufrage constitue à la fois une occasion d'enrichissement frauduleux ou, au contraire, une opportunité d'avancement dans l'estime des autorités coloniales.

La description la plus élaborée d'un naufrage mentionné sur le site web de Charles Vianney Campeau est certes celle consacrée au navire le Saint-Joseph de Québec. Ce bâtiment jaugeant entre 116 et 160 tonneaux, navigue dans un triangle Atlantique s'étendant à la Martinique et à Bordeaux. C'est pourtant à l'Île d'Anticosti ${ }^{30}$ qu'il $^{\prime}$

2513 novembre 1680, Lettre de Duchesneau au ministre, BAC-MG1-C11A, bobine C2375, vol. 5 , f $164-165 \mathrm{v}$.

26 Marcel Moussette et Gregory A. Waselkov, Archéologie de l'Amérique Coloniale française, Montréal, Lévesque Éditeur, Réflexion, 2013, p. 321. Voir aussi Lafrance, Les épaves du Saint-Laurent, pp. 129-131, incluant une carte montrant le lieu du naufrage, p. 132.

27 Site web de Charles Vianney Campeau. Navire ${ }^{\circ}$. 1808. 1723. .

2825 octobre 1729, Lettre de Hocquart au ministre, BAC-MG1-C11A, bobine C2389, vol. 51, f 268-269. Cet épisode a retenu l'attention de Thomas Wien. Ce naufrage se déroule à une cinquantaine de kilomètres en aval de Québec. Il explique que les autorités de Québec édifièrent cet événement en véritable sauvetage exemplaire. Tous les participants, excepté le pilote du vaisseau, se démarquèrent. On parle néanmoins « d'erreur de jugement, de débarquement dans la confusion, de pillage d'effets par les soldats avec la complicité des matelots ", etc. Wien se demande si l'on ne cherche pas justement à faire oublier le naufrage du Chameau. Thomas Wien, «Rex in fabula : travailler l'inquiétude dans la correspondance adressée aux autorités métropolitaines depuis le Canada (1700-1760) », Outre-mer, tome 96, $\mathrm{n}^{\mathrm{o}} 362-363,1^{\mathrm{er}}$ semestre 2009, pp. 65-85. Voir aussi Lafrance, Les épaves du Saint-Laurent, pp. 81-88, incluant une gravure relatant l'événement.

29 Banks, Chasing Empire, 74.

30 Selon Proulx, en 1736, la Renommé s'échoue elle-aussi sur l'île d'Anticosti. Proulx, Entre France et Nouvelle-France, p. 73. 
s'échoue lors d'un voyage devant le conduire jusqu'au Labrador. Le rapport de ces événements stipule que ce navire parti de Bordeaux en juillet, passe le Cap de Ré (Ray?) (Pointe sud-est de Terre-Neuve) en août alors qu'il est « pris d'un coup de vent de sud sud-est avec brume, pluie, tonnerre et éclairs ", le forçant à s'échouer sur l'île d'Anticosti vers $6 \mathrm{~h}$ du matin. Dès lors, la chaloupe est mise à la mer et les mâts sont coupés afin de " soulager le navire et sauver les biens ». Ces marchandises sont ensuite entreposées sur la rive où on en dresse un inventaire. Mais les vivres restantes se résument à « une barrique et demi de pain, un baril de bœuf, un sac de pois, une demi barrique de morue », en bonne partie gâtée par l'eau salée. C'est là que le capitaine et les officiers proposent aux vingt-quatre hommes d'équipage de diviser le groupe en deux; l'un gardera le navire et sa cargaison alors que l'autre ira chercher des secours avec la chaloupe. Ce n'est cependant que le 7 septembre que la chaloupe part en direction du Fort Beauharnois à Mingan, avec onze hommes à bord. Une fois sur place, ce petit groupe a la chance de voir arriver une goélette commandée par Charles Le Cour en provenance de la Baie des Châteaux. Dans le cadre de sa déclaration à Québec, Damour rappelle qu'il a fait assurer le navire et sa cargaison à Bordeaux et qu'il en fait maintenant «l'abandon aux assureurs ». Il s'engage de transporter les marchandises sauvées dans un magasin du roi. Dans une correspondance d'octobre 1742, Beauharnois et Hocquart mentionnent au ministre qu'ils ont envoyé un bateau de Québec pour ramener les douze hommes restés à Anticosti et aussi récupérer la cargaison. Hocquart écrit également au ministre que ce naufrage a fait chuter de « 5000 à 6000 livres les revenus provenant des droits d'entrée sur le vin ». Par conséquence, cette rareté a fait monter le prix du vin à 140 livres la barrique! ${ }^{31}$

En plus du Saint-Joseph, un autre navire voyageant entre Québec et Bordeaux est l'Heureux (144 à 150 tonneaux). Sous les ordres du capitaine Jean Darracq, il part de Bordeaux en mai et ensuite de l'île d'Aix en juin pour retourner à Québec. Mais il coule à Baie Rouge près du Labrador le 20 août. La cargaison a là-aussi été assurée à Bordeaux pour 27.000 livres par Nicolas Beaujon, possiblement apparenté à Jean Beaujon, l'un des deux propriétaires du navire avec Pierre Trottier Desauniers. À noter que ce bâtiment quitte l'île d'Aix dans le fameux convoi du Duc d'Anville ${ }^{32}$ mais en juillet, de gros vents séparent ce navire du reste du convoi. Il navigue alors dans les " brouillards forts épais » durant cinquante-huit jours, avant de heurter des rochers à Baie Rouge le 20 août. Le capitaine et son équipage réussissent à atteindre la rive dans un " dériveur ». Le commandant à Baie Rouge, Monsieur Desruisseau, leur prête des chaloupes pour débarquer l'eau-de-vie qui est ensuite vendue à Québec avec un bénéfice de 3231 livres. Il existe un contrat d'abandon pour ce navire, daté du 29 décembre 1746, rédigé à Bordeaux à la demande de Jean Beaujon ${ }^{33}$. Cet épisode illustre fort bien qu'en plus des assurances, les dommages peuvent être compensés par la vente de la cargaison après les opérations de sauvetage.

31 Site web de Charles Vianney Campeau, 1742, Navire n 2383, Le Saint-Joseph.

32 À ce sujet, le meilleur ouvrage à ce jour demeure celui de Jim Pritchard, Anatomy of a Naval Disaster : The 1746 French Expedition to North America, Montréal et Kingston, McGillQueen's University Press, 1995.

33 Site web de Charles Vianney Campeau, 1746, Navire nº 2503, L'Heureux. 
Les extraits ci-haut illustrent encore une fois que la sauvegarde des cargaisons et l'entretient des équipages rescapés, imposent des dépenses non négligeables aux administrateurs coloniaux. Il est à parier qu'il ne leur ait pas toujours facile de convaincre des riverains de s'investir dans ces opérations sans promesse de récompenses substantielles. Également, la perte de cargaisons précieuses telles le vin, peut provoquer une augmentation substantielle des prix à la consommation.

\section{Dans les parages de l'île Royale!}

En plus des naufrages et incidents survenant à proximité des côtes européennes, dans l'Atlantique ou dans le Saint-Laurent, plusieurs pertes de navires et de chaloupes de pêche sont régulièrement signalées dans les parages de l'île Royale ${ }^{34}$. Pour cette colonie, soulignons d'abord le naufrage d'un navire dont le souvenir persiste de nos jours. Ainsi, en 1725, survient le naufrage du navire le Chameau ${ }^{35}$ alors que 109 hommes de troupe périssent avec la cargaison. Madame Duvivier ${ }^{36}$, pour sa part, se dit alors rassurée d'apprendre que son mari ne s'était pas embarqué sur ce navire. Pour remplacer ces pertes, le roi envoie l'Éléphant l'année suivante, espérant ainsi que « le retardement des fonds et effets » perdus sur le Chameau «n'a point dérangé le service de la colonie ». Ce sont également les mesures prises par l'Intendant qui permettent de trouver des vivres et « le petit habillement » pour les troupes ${ }^{37}$. Des chiffres datant de 1728 permettent de constater que les pertes découlant du naufrage du Chameau se chiffrent à environ 52006 livres tournois, soit environ 29000 livres en espèces et un peu plus de 23000 livres en marchandises et munitions ${ }^{38}$.

L'été 1731 s'avère particulièrement éprouvant pour cette colonie en termes d'incidents maritimes. Les officiers de l'amirauté de Louisbourg énumèrent dix-sept

34 Si l'on se rapporte au tout début du $17^{\text {e }}$ siècle, en 1629 , il ne fait aucun doute que le naufrage du navire transportant les missionnaires Charles Lalemant, Philibert Noyrot et leurs collègues au large du Cap-Breton demeure un évènement symbolique important des dangers de la navigation dans ces eaux. On déplore alors quatorze victimes. Rainer K. Baehre, Outrageous Seas. Shipwreck and Survival in the Waters off Newfoundland, 1583-1893, Montréal et Kingston, McGill-Queen's University Press, 1999, p. 69. À noter que cet ouvrage relate surtout des naufrages de navires anglo-saxons du $19^{\mathrm{e}}$ siècle.

35 Le Chameau est un navire royal partant de Rochefort au début juillet, sous le commandement du capitaine Saint James. Il coule le 28 août au large de Louisbourg avec son équipage de 250 hommes. Site web de Charles Vianney Campeau, Navire $\mathrm{n}^{\text {ono }} 1877$, Le Chameau.

36 On parle ici de François Du Pont Duvivier, commerçant et militaire à l'île Royale. Il sera tour à tour lieutenant, aide-major et capitaine. T.A. Crowley et Bernard Pothier, « Du Pont Duvivier, François (1705-1776) », Dictionnaire biographique du Canada, vol. 4.

3720 mai 1726, Lettre de Bégon au ministre, BAC-MG1-C11A, bobine C2388, vol. 48, f $207-$ 209. On peut consulter les Arrêts, édits et ordonnances de la Marine pour se familiariser avec les instructions acheminées aux commis des fermes des bureaux maritimes au sujet des échouements et naufrages. 28 mars 1726, BAC-MG1-Série 3S2, Fonds du Service historique de la Marine à Vincennes, vol. 21, dossier 341, 10 pages.

3825 mai 1728, État de la situation du Sieur Lanouillier, BAC-MG1-C11A, bobine C2406, vol. 113, partie 2, pp. 256-258. 
embarcations de toutes catégories ayant essuyées de lourdes pertes ou tout bonnement disparues. Il s'agit de neuf chaloupes de pêche, de trois goélettes de pêche, d'un brigantin et de quatre navires. D'abord, un bâtiment de Saint-Malo coule en allant faire la pêche à l'île Saint-Jean. Toute la cargaison est perdue, sauf le sel. Un autre bâtiment, revenant de l'île Saint-Jean, est victime d'une tempête qui le garde en mer près d'une dizaine de jours avant qu'il ne se brise du côté de Canso. Son équipage de neuf hommes péris au complet, à l'exception d'un matelot espagnol qui s'agrippe à des débris pendant près de 16 heures avant d'être rescapé par une chaloupe anglaise. L'équipage d'un bâtiment de Nantes est plus chanceux puisque c'est seulement la cargaison, à l'exception de quelques agrès et apparaux, qui sont endommagée. Durant cette même tempête, neuf bateaux de pêche de l'île Royale sont jetés à la côte; deux sont « entièrement brisés 》 et un autre, chargé de morues, coule dans la rade. À cela s'ajoute la perte de petites chaloupes dont le nombre demeure inconnue. Finalement, un navire de vivres venant du Canada coule sur les « battures » de l'île Saint-Jean. Un autre équipage chanceux est celui d'un brigantin en provenance de la Martinique et se rendant à Québec, alors que dans l'anse du Cap des Rosiers, « à l'entrée de la Rivière du Canada » (fleuve SaintLaurent), un naufrage lui fait perdre toute la cargaison mais l'équipage de soixante-dix hommes est sauf. Cinq d'entre eux décident alors de se rendre à Québec à pieds, tandis que les autres sont embarqués par une goélette de l'Île Royale en relâche à Gaspé. Durant cette même tempête, trois " bateaux pêcheurs » de l'île Royale sombrent en revenant des bancs de pêche. Il s'agit vraisemblablement de goélettes ${ }^{39}$.

D'autres « coup de vent du sud-ouest, en forme d'ouragan », provoquent l'échouement de plusieurs petits bâtiments. Entre autres, un navire ayant chargé à Bordeaux et avec encore la moitié de sa cargaison, est jeté sur les rochers, « au pied du fanal » de Louisbourg et mis en pièces ${ }^{40}$. On signale aussi un brigantin du Canada, chargé en partie de vivres, et une goélette, tous deux coulés lors de la même tempête. Le drame frappe aussi une chaloupe d'habitants avec un équipage de cinq hommes. Chargée de vivres, de sel et d'ustensiles de pêche, elle était sortie cette même nuit pour aller débuter sa pêche d'automne au havre du Saint-Esprit ${ }^{41}$.

En 1739, Antoine Sabatier ${ }^{42}$, contrôleur à l'île Royale, rapporte d'autres incidents maritimes s'étant produits récemment. Il évoque tour à tour la perte du Saint-Jean ${ }^{43}$, des

398 janvier 1732, Les officiers de l'amirauté de Louisbourg au ministre, BAC-MG1-C11B, bobine C9293, vol. 13, pp. 86-89.

40 Le fanal de Louisbourg est inauguré en 1732 dans l'espoir de diminuer le nombre d'accidents survenus à plusieurs bâtiments à l'approche du havre de cette capitale coloniale de l'île Royale. Le 26 juin 1732, le Conseil d'État à Compiègne émet un arrêt sur les droits à être perçus sur les vaisseaux pour « l'entretien de ce fanal, effectif en avril 1733 ». Site web de Charles Vianney Campeau. .

4122 décembre 1735, Monsieur le Vasseur, écrivain, et monsieur de La Forest, île Royale, Terrible ouragan du 11 novembre, BAC-MG1-C11B, bobine C9293, vol. 17, pp. 129-132.

42 Sur cet administrateur, voir T.A. Crowley, « Sabatier, Antoine », Dictionnaire biographique du Canada, Vol. III (1741-1770).

43 Le Saint-Jean, d'Olonne, est sous les ordres du capitaine Charles Texier. Il part de Bordeaux 
Sables d'Olonne, naufragé près de Canso, la Madeleine de Saint-Jean-de-Luz et la Licorne de Morlais. La Madeleine se retrouve prise dans les glaces et jetée à la côte en ne sauvant que l'équipage et quelques agrès, alors que la Licorne est malmenée par les glaces à l'entrée du port. Ce bâtiment fini écrasé, en ne sauvant que cinq des douze hommes d'équipage et le capitaine D'hiverbeau figure parmi les «malheureux » disparus $^{44}$.

Il y a ici lieu d'ouvrir une parenthèse pour mentionner l'accueil des matelots naufragés à l'hôpital royal de l'île Royale. Par exemple, ceux du navire du roi le Héros, qui sont déclarés « dégradés, naufragés, malades et insolvables dans la colonie ». Ils sont donc soignés et nourris pendant les trois derniers mois de 1734 au coût de 884 livres ${ }^{45}$. Il semble que le navire le Rubis connaît également des avaries puisque trente-neuf matelots de cet équipage passent eux-aussi quelques mois à l'hôpital royal la même année, au coût de 622 livres $^{46}$.

À l'occasion, des administrateurs de l'Île Royale attribuent certains incidents maritimes au manque de connaissance ou de compétence des navigateurs. Par exemple, en 1743, le procureur du roi à l'Île Royale déplore les fréquents « naufrages et pertes de bâtiments tant des pêcheurs que de ceux qui font le commerce de port en port » ou cabotage. Il dénonce ce qu'il estime être de l' "impéritie » ou imprudence de ceux commandant ces navires. Il mentionne deux lacunes chez ces derniers soit un manque de maîtrise de la navigation et une méconnaissance du littoral de l'île Royale. Pourquoi ne pas leur imposer des « attestations de deux années de navigation » dans cette colonie, d'être âgé d'au moins vingt-cinq ans et d'avoir été soumis à un examen administré par deux « anciens capitaines » nommés par l'amirauté de l'île Royale ${ }^{47}$ ? Ce même automne,

pour l'Acadie mais s'échoue et est déclaré perdu le 23 janvier 1739. Il avait été assuré le 11 septembre 1738 par Charles de La Richardière Petiot. Site web de Charles Vianney Campeau, 1738, Navire n no 2220, Le Saint-Jean.

443 août 1739, Monsieur Sabatier, contrôleur à l'île Royale, au ministre, BAC-MG1-C11B, bobine C9294, vol. 21, pp. 155-161.

453 octobre 1735, Extrait des registres de l'hôpital royal de Louisbourg, BAC-MG1-C11B, bobine F145, vol. 16, f 109-110. Pour d'autres informations sur des naufrages, voir un document du 9 juin 1733, Lettre de Maurepas répondant à des rapports, BAC-MG6-A2, bobine C9184, vol. 6110, p. 25. En 1735, le roi émet une déclaration concernant les naufrages maritimes. 15 juin 1735, Fonds du Service historique de la Marine, Série 3S2, vol. 40, dossier 396.

463 octobre 1735, Extrait des registres de l'hôpital royal de Louisbourg, BAC-MG1-C11B, bobine F146, vol. 17, f 113-114v. D'ailleurs, le 15 août 1732, un navire de Louisbourg, la Revanche, (110 tonneaux), dont le maître est Michel Rodrigue, part de ce port à destination de Québec pour y transporter les soldats et prisonniers du Rubis, hospitalisés à Louisbourg durant un certain temps. Comble de malchance, le navire fait naufrage à Nigoniche. Deux hommes se noient dont le soldat Julien Guineau et un prisonnier. Site web de Charles Vianney Campeau, 1735, Navire $\mathrm{n}^{\circ}$ 2035, La Revanche.

478 avril au 24 septembre 1743, Requête du procureur du roi de Louisbourg, BAC-MG6-A2, bobine C9184, vol. 6114, p. 165-168. Mais il arrive aussi que des tensions surgissent entre fonctionnaires royaux, jaloux de leurs prérogatives respectives. Par exemple, en 1741, on signale des tiraillements entre le Directeur du Domaine d'Occident, le receveur et Monsieur 
on relate des naufrages survenant à Terre-Neuve et à Gaspé. Entre autres navires mentionnés; les Trois Amis et l'Amazone. On attribue ces pertes aux ravages des glaces du printemps ${ }^{48}$.

Toute cette question de remboursements de dépenses attribuables aux sauvetages peut également susciter de la méfiance chez certains administrateurs. Sans témoignages pour corroborer la version d'un capitaine prétendant avoir porté secours à des naufragés, comment être certain de ne pas être victime de fraude? Un bon exemple de ce genre de situation survient en 1747. Le président du Conseil de Marine de France s'adresse aux commissaires des Échanges à Londres pour contrevérifier des faits relatifs à des naufragés de l'île de Sable. On désire savoir si John Gorham a réellement fait pour eux les dépenses dont il réclame le remboursement à la Marine ${ }^{49}$. Des frais de transport sont également payés pour des matelots naufragés à la Martinique mais arrivés à Louisbourg. De là, ils s'embarquent sur la flute du roi le Caméléon, en direction de Québec ${ }^{50}$. Mais comble de malheur, ce navire fait naufrage à son tour au Sault de la Chaudière, près de Québec. Également, les matelots du navire le Saint-Joseph de Saint-Malo, naufragé à Gaspé, se trouvent sur le Caméléon ${ }^{51}$.

Les incidents de 1754 se retrouvent d'ailleurs dans un « cahier des conduites payées » à La Rochelle, aux équipages et passagers de vaisseaux pris par l'ennemi ou naufragés, de même qu'à des personnes libérées des prisons d'Angleterre. Il y figure les mêmes noms d'habitants et de soldats du Canada et de l'île Royale, de même que de membres d'équipage de quatorze navires, incluant le Caméléon et le Saint-Joseph ${ }^{52}$. On pourrait donner d'autres exemples de frais encourus pour des naufragés, cette fois datés de 1760, découlant d'incidents maritimes aux Açores. Ce sont alors les sieurs De Cous, de Fiedmont, de Lusignan et de Léry qui demandent des remboursements qui leur sont effectivement accordés par la Marine ${ }^{53}$.

l'Amiral à l'occasion de naufrages et d'échouements, 1741, Mémoire sur le Domaine d'Occident au Canada, BAC-MG1-C11A. À noter, en 1742, la parution de l'ouvrage d'Emmanuel Crespel, dont le titre en anglais est Voyages of Rev. Father Crespel in Canada and his shipwreck while returning to France, Frankfort-on-the Meyn, Louis Crespel, 1742.

4821 octobre 1743, Lettre de Guillot au ministre, BAC-MG2-B3, bobine C11620, vol. 415, f $84-85 \mathrm{v}$.

4930 septembre 1747, le président du Conseil de Marine à messieurs les commissaires des Échanges à Londres, BAC-MG1-B, bobine F289, vol. 86, f 146.

50 Le Caméléon est une flute du roi, dont le capitaine est Foucault. Le navire parti de Rochefort à destination de Québec, fait naufrage au Sault de la rivière Chaudière, avant le 8 octobre 1754. Site web de Charles Vianney Campeau, 1754, Navire n 2947, Caméléon.

51 Septembre 1754, Naufragés, BAC-MG6-A2, bobine C9185, vol. 6121, pp. 3-10.

52 1748-1757, Extrait du cahier des conduites payées à La Rochelle, BAC-MG6-A2, bobine C9182, vol. 252, pp. 2-15. On peut aussi se référer à des extraits du cahier des conduites de matelots naufragés, venant des prisons d'Angleterre, de Québec et de l'île Royale daté de 1759, Janvier-avril 1759, BAC-MG6-A2, bobine F469, vol. 255, 29 pages.

5314 septembre 1760, Président du Conseil de la Marine à Monsieur Ranché, Certificats, BACMG1-B, bobine C15663, vol. 112, f 221. 
À la toute fin du Régime français au Canada, un naufrage somme toute assez célèbre est celui de l'Auguste, ayant sombré au large des côtes du Cap-Breton le 16 novembre 1761. Il part de Québec le 15 octobre avec à son bord le Chevalier de SaintLuc de la Corne. Le navire commence par perdre une de ses ancres au mouillage de l'Îleaux-Coudre et le feu se déclare ensuite « à trois reprises dans la cambuse» avant la fin de l'aventure ${ }^{54}$. On ignore si des matelots ayant pu survivre à ce naufrage, purent bénéficier ou non de dédommagement de la part de la Marine. Effectivement, en 1762, l'administration de la Marine doit veiller à verser la solde d'officiers naufragés ou restés au Canada après la défaite ${ }^{55}$.

\section{Saint-Pierre et Miquelon}

Suite au Traité de Paris de 1763, la France ne conserve que les îles Saint-Pierre et Miquelon à titre de colonie dans le Golfe Saint-Laurent ${ }^{56}$. Là-aussi, au cours des années menant à la Révolution française et aux guerres napoléoniennes, de nombreux naufrages viennent perturber l'équilibre budgétaire de l'administration locale. Ainsi, dès le printemps 1769 , une tempête importante provoque le naufrage de trois navires sur les côtes de Terre-Neuve et la colonie naissante de Saint-Pierre et Miquelon doit accueillir 101 personnes $^{57}$. Ces dernières sont amenées dans l'archipel sur des chaloupes anglaises et la problématique des provisions rend la situation critique pour le gouverneur d'Angeac $^{58}$. Sa priorité consiste donc à trouver des occasions pour faire repasser ces naufragés en France le plus vite possible. On sait qu'en juin, les seize derniers naufragés s'embarquent pour La Rochelle $\mathrm{e}^{59}$.

À Saint-Pierre et Miquelon, les déchaînements occasionnels de la mer ne s'embarrassent guère des nouvelles réglementations royales sur la sécurité maritime et la sauvegarde des intérêts des armateurs. Ainsi, en 1775, les autorités françaises rapportent

54 Encyclopédie de l'histoire du Québec. http://faculty.mariapolis.edu/c. Juin 2006.

5526 octobre 1762, Président du Conseil de la Marine à Monsieur Périchon, BAC-MG1-B, bobine F322, vol. 115, f 276. À la veille du Traité de Paris de 1763, Choiseul achemine à l'Amirauté de Guyenne les instructions nécessaires à l'exécution des ordonnances et règlements sur les naufrages, 29 avril 1762, Lettre de Choiseul aux officiers de l'Amirauté de Guyenne.

56 Pour les naufrages survenus dans les parages de Saint-Pierre et Miquelon, mais à compter de la fin du $19^{\mathrm{e}}$ siècle surtout, voir Joseph Lehuenen, Jean-Pierre Andrieux, Naufrages! Histoire illustrée des désastres maritimes aux îles Saint-Pierre et Miquelon, Beamsville, Ontario: W.F. Rannie, 1977.

57 Sur la question des naufrages et autres pertes de vie dans l'industrie française des pêches terre-neuviennes, surtout au $19^{\mathrm{e}}$ siècle, voir Lucien Marie, "Mourir à Terre-Neuve ", dans Éric Barré (dir.), Troisièmes Journées d'Histoire de la Grande Pêche (Granville, 18-19 mars 2005), Saint-Lô : Société d'Archéologie et d'Histoire de la Manche, 2007, pp. 168-200.

5822 mai 1769, Lettre de Monsieur d'Angeac à Monsieur le duc de Praslin, ministre de la Marine, BAC-MG6-A17.

595 juin 1769, Lettre de Monsieur de Beaudéduit, ordonnateur à Saint-Pierre et Miquelon, à Monsieur le duc de Praslin, ministre de la Marine, BAC-MG1-C12, bobine F569, vol. 3, f 30-30v. 
le sauvetage de naufragés anglais en route vers Québec. Rappelons aussi que dans la nuit du 18 au 19 novembre 1774, surviennent les naufrages du Loup Marin et de La Vierge ${ }^{60}$. Cette tempête aux proportions légendaires, a grandement suscité l'intérêt des chercheurs depuis les années 1930. Bien que les opinions divergent en ce qui a trait au nombre de victimes, le baron de l'Espérance estime que des quarante goélettes françaises se trouvant alors sur les bancs, vingt-deux furent perdues, presque toutes appartenant aux habitants des îles, sans compter la perte de cinquante chaloupes, la destruction de nombreux chafauds et la mort de 400 hommes. Aux îles mêmes, dix-huit goélettes, vingt-et-une chaloupes et trois chafauds avaient été brisés ou submergés ${ }^{61}$. Pour sa part, le gouverneur de Terre-Neuve Robert Duff, parle de trois navires faisant naufrage, entraînant la perte de 9400 quintaux de morue, de trente tonnes d'huile et de la destruction de plusieurs embarcations de toutes tailles. Il estime qu'à l'île Miquelon, une vingtaine de chaloupes disparaissent et neuf navires font naufrage. Les équipages des goélettes de Miquelon comptent de cinq à neuf hommes alors que ceux des vaisseaux venant de France en compte dix-huit en moyenne ${ }^{62}$. Durant les semaines qui suivent, les rumeurs veulent que des pêcheurs de St-John's trouvent de vingt à trente victimes dans leurs filets ${ }^{63}$.

Quelques années plus tard, entre le $1^{\text {er }}$ et le 16 octobre 1783, survient une série de violentes tempêtes provoquant le naufrage de plusieurs navires de l'île Miquelon ${ }^{64}$. L'un des cas les mieux documentés en termes de naufrages est sans doute celui du navire Les Trois Sceurs, survenu le 31 mai 1784, non loin de Cape Race sur la côte de Terre-Neuve. Le rapport de l'événement s'appuie entièrement sur le témoignage du capitaine le sieur Monbart, à son arrivée dans le port de Saint-Pierre. Ses déclarations sont d'ailleurs confirmées par l'équipage et les passagers. À son départ de Saint-Malo, ce bâtiment compte 165 passagers, tant hommes, femmes qu'enfants. Trois passagers décèdent durant la traversée et au moment du naufrage, on dénombre 162 passagers et trente hommes d'équipage soit 192 personnes au total. Il semble que le naufrage survint près d'un rocher de « 20 pieds à 26 pieds de haut presqu'à pic et distant d'environ 16 lieues de la Baye des Trépassés (Trepassey Bay) ${ }^{65}$.»

6019 juin 1775, Lettre du baron de l'Espérance, BAC-MG1-C12, bobine F569, vol. 4, f 178182. Voir aussi 1774-1775, novembre-janvier, Amirauté du Havre, Échouages et naufrages, 216 B.P. BAC-MG6-A9, bobine F1700, vol. 310.

61 Alan Ruffman, " The Multidisciplinary Rediscovery and Tracking of The Great Newfoundland and Saint-Pierre et Miquelon Hurricane of September 1775 », The Northern Mariner / Le marin du nord, VI, no 3, July 1996, p. 17.

62 Anne E. Stevens and Michael Staveley, « The Great Newfoundland Storm of 12 September $1775 »$, Bulletin of Seismological Society of America, vol. 81, n 4, August 1991, pp. 14001401.

63 David Liverman, Norm Catto, Martin Batterson, « Geological Hazards and Disasters in StJohn's », Newfoundland and Labrador Studies, vol. 21, n 1, 2006, p. 1.

6427 octobre 1783, Lettre de messieurs le baron de l'Espérance, gouverneur, et de Malherbe, ordonnateur, à Monsieur de Castries, ministre de la Marine, BAC-MG1-C12, bobine 571, vol. 8, f 19-19v.

6529 juillet 1784, Lettre de Malherbe, commissaire ordonnateur, au ministre, BAC-MG1-G1, bobine F698, vol. 467, 7 pages. 
Avant que le navire ne sombre, le capitaine et ses hommes ont juste le temps de récupérer des biscuits et quelques voiles de rechange. Tous les rescapés réussissent à se hisser sur le rocher où s'élève une tente assez longue pour abriter les survivants « de la brume très épaisse qui fut suivie d'une pluie très abondante qui dura plus de deux jours consécutifs ». Un détachement de quelques hommes, dont le capitaine, atteignent la Baie des Trépassés (côte de Terre-Neuve) après trois jours de marche. Dans ce port, après avoir fretté des bateaux de pêche avec des vivres, ce n'est qu'au $10^{\mathrm{e}}$ jour d'attente que le reste des « infortunés » sont recueillis. Durant ce temps, ils doivent se contenter d'une ration quotidienne de quatre onces de biscuits, d'herbages et de racines. Les bateaux de pêche en question amènent les rescapés à St-John's, alors que le capitaine frète une goélette pour se rapporter aux autorités de Saint-Pierre, accompagné de vingt-cinq hommes d'équipage et de huit passagers. La goélette arrive à Saint-Pierre le 15 juin. Par la suite, le baron de l'Espérance donne l'ordre à monsieur d'Alpharan, commandant de la corvette royale la Belette, de se rendre à St-John's pour en ramener le plus de rescapés possible. La lettre adressée au commandant anglais de la place s'accompagne de quelques présents, en guise de reconnaissance pour l'aide apportée aux naufragés français. D'Alpharan y est fort bien accueilli et réussit à embaucher une goélette de pêche afin de l'assister dans le rapatriement à Saint-Pierre de tous les rescapés, soit audelà d'une centaine. Ces derniers suscitent la pitié des autorités à leur arrivée à SaintPierre; « dénués entièrement de hardes n'ayant que celles qu'ils avaient à bord et sans le moindre rechange ayant même perdu leurs lits avec leurs effets et ustensiles de ménage ». Les autorités font preuve d'ingéniosité pour leur venir en aide en organisant une fête qu'ils appellent « la réjouissance de la Paix » et distribuent des effets aux naufragés pour une somme équivalente à 2.400 livres! Il fallait aussi rembourser les dépenses découlant de l'assistance des Anglais soit 446 livres. Au total, les dépenses atteignent 7.229 livres!

Du côté des autorités de Saint-Pierre, on déplore aussi la perte de matériel destiné à l'hôpital de l'archipel dont des lits, des draps et autres ustensiles. En attendant du secours en provenance des premiers navires en partance de Saint-Malo, Granville ou Bayonne au printemps 1785, Malherbe prie monsieur de l'Etourbe, Consul général de France à Boston, de lui faire parvenir 300 livres de linges à pansement.

De juin à septembre 1784, l'impact financier de la présence de ces réfugiés à Saint-Pierre laisse des traces. Dès juin, on achemine à la cours un premier bilan des vivres et rafraîchissements que la corvette la Belette a acheté à St-John's pour répondre aux besoins des 169 naufragés ${ }^{66}$. En plus des vivres et des sommes qui leur sont remises, on leur accorde également plusieurs cordes de bois pour se chauffer et cuisiner ${ }^{67}$.

Ces demandes accrues d'approvisionnements et de rapports de naufrages se maintiennent en 1786 et 1790. À l'automne 1786, le Commandant Danseville demande à

6623 juin 1786, État des vivres et rafraîchissements, BAC-MG1-C12, bobine F571, vol. 8, f $65 \mathrm{v}$.

6719 septembre 1784, Lettre de messieurs le baron de l'Espérance et Malherbe à monsieur de Castries, ministre de la marine, BAC-MG1-C1, bobine F571, vol. 8, f 79v-80. Le lecteur peut aussi se référer à un autre document portant sur les paiements des bâtiments naufragés à Miquelon. Vers 1784, BAC-MG1-DEF, bobine F589, vol. 2, 76/90, items 8 pages. 
Castries, ministre de la Marine, d'envoyer davantage d'approvisionnements en raison des naufragés des navires se perdant près des côtes de la colonie. Il préfère du lard salé puisque le « bœuf se détériore trop rapidement ${ }^{68}$. » Il semble qu'il s'agisse surtout de bateaux étrangers et il répète les conséquences en découlant sur « l'état des vivres dans la colonie $^{69} . »$

Fenêtre quantitative sur les incidents maritimes du $18^{e}$ siècle entre France et Nouvelle-France

Afin d'étayer davantage l'aspect quantitatif de notre recherche, nous avons exploré l'impressionnant site web de Charles Vianney Campeau pour déterminer sommairement le nombre de navires endommagés ou perdus entre 1700 et $1760^{70}$. Et ce, autant en temps de guerre que de paix. Tel qu'expliqué par Laurent Huet, ce site présente une " liste des navires français arrivés sur les côtes canadiennes par année, avec le tonnage, le nom de l'armateur, du propriétaire, du capitaine, la destination du départ ainsi que le retour ${ }^{71}$. » À cela s'ajoute des renseignements « sur l'équipage, les passagers et ce qu'il advient par la suite des rescapés et de la cargaison » en cas d'incident maritime. Précisons cependant que ce ne sont pas tous les navires pour lesquels on dispose de ces informations. De plus, pour le tonnage, on a souvent affaire à une approximation entre deux chiffres. En dépit de ces réserves, le travail encyclopédique de Vianney Campeau répond à un vide historiographique. D'ailleurs, au moment où il publie son ouvrage en 1996, Pierre Camu estime ne disposer que « de quelques données fragmentaires sur le nombre de collisions, d'échouages, de naufrages, de pertes complètes et de dommages subis par les navires sur le Saint-Laurent » durant le Régime français. Il n'a d'ailleurs aucune «données sur les navires perdus en $\mathrm{mer}^{72}$. » Précisons toutefois que Camu n'aspirait pas à estimer le nombre d'incidents maritimes survenus dans le golfe à cette époque. Dans notre cas, rappelons que notre compilation respecte les trois entités géographiques mentionnées en introduction soit l'Atlantique (en pleine mer ou à proximité des côtes européennes), dans l'entourage de l'île Royale et finalement la route

6826 novembre 1786, Lettre de Danseville à de Castries, ministre de la Marine, BAC-MG1C12, bobine F571, vol. 8, f 96v-97.

6930 décembre 1786, Lettre de Danseville à de Castries, BAC-MG1-C12, bobine F571, vol. 8, f 97. Pour un rapport d'incident maritime particulièrement élaboré, le lecteur peut se référer à l'épisode du navire la Confiance (250 tonneaux). Il transportait du tabac en partance de Georgetown (Géorgie) le 24 mai et fut victime d'un incident au Havre (France) le 13 juillet 1790. Juillet-août 1790, Amirauté du Havre, Échouages et naufrages, BAC-MG6-A9, bobine F1700, vol. 310, 38 pièces.

70 À noter que Mario Mimault s'est également servi de ce site web dans son article « Du golfe Saint-Laurent aux côtes de Bretagne et de Normandie (1713-1760) », Revue d'histoire de l'Amérique Française, Vol. 67, n ${ }^{\text {ono }}$ 1, été 2013, pp. 5-32.

71 Laurent Huet, «L'économie du naufrage », p. 35.

72 Pierre Camu, Le Saint-Laurent et les Grands Lacs au temps de la voile 1608-1850, Cahiers du Québec, HMH : Collection géographie. Ville La Salle, 1996, p. 61. 
du Canada en remontant le fleuve et le long de la côte jusqu'au Labrador ${ }^{73}$.

C'est ainsi que nous en arrivons à un grand total de 240 incidents dont soixantequinze en temps de paix et 165 (soixante-huit pourcent) en temps de guerre. Les qualificatifs employés pour désigner un incident maritime sont variables. Par exemple, dans le cas des incidents non provoqués par des actes de guerre, on dénombre trente-cinq mentions où prédominent trois termes que sont échouements et naufrages avec vingt occurrences et coulés avec quinze. On pourrait y ajouter une foule d'autres qualificatifs tels que perdue (cinq occurrences), pris dans les glaces (4), saisie (4), chaviré, disparue, condamnée, explosé, détruit ou encore brûlé.

De 1700 à 1759, il est rare que l'on note plus d'un ou deux incidents par année. En fait, durant cette période, il y a trente-huit années sur cinquante-neuf où l'on retrouve au moins une ou plusieurs mentions pour une moyenne globale d'environ deux par année. Trois années sont particulièrement notoires soit 1740, 1750 et 1759 avec sept incidents par année soit un total de vingt-et-un sur soixante-quinze ou vingt-huit pourcent. Plusieurs ports d'attache sont mentionnés soit cinquante-cinq au total, dont la Rochelle et Bordeaux à dix-sept reprises chacun et Québec à neuf occasions. Le fleuve Saint-Laurent semble avoir été le lieu d'au moins vingt incidents, alors que cinq se produisent à l'île d'Anticosti et trois au Cap-Breton. À huit reprises on mentionne des lieux le long des côtes françaises. On pourrait y ajouter le Labrador (1) et Saint-Domingue (2).

Selon Ruette d'Auteuil, la guerre de Succession d'Espagne est effectivement l'une des principales causes d'incidents maritimes et de « pertes que le Canada a souffert par mer ». Il précise sa pensée en disant qu'un bon nombre de navires ont été perdus « faute de les avoir fait partir en flotte et de leur avoir donné des convois ${ }^{74}$. » Également, il attribue un certain nombre de naufrages au fait qu'on a laissé des navires « partir trop tard du Canada ", c'est-à-dire vers l'automne. Il estime que depuis 1690, les pertes essuyées en raison de naufrages se chiffrent à trois millions et demi de livres tournois ${ }^{75}$.

Durant les trois guerres coloniales du $18^{\text {e }}$ siècle, sur les 165 pertes de navires français, 116 (soixante-dix pourcent) surviennent durant la Guerre de Sept Ans et surtout en 1757 et 1758 avec respectivement trente-huit et quarante-quatre pertes ${ }^{76}$. À noter que c'est au printemps (avril-mai) que se manifestent des pertes en cascades du côté français avec quatorze en 1757 et quinze en 1758, sans oublier les quatre navires perdus lors de la bataille de la Ristigouche en 1760 soit le Marquis de Malause, le Bienfaisant, L'Aurore et

73 Pour une description plus précise des étapes à franchir dans la remontée du Saint-Laurent vers Québec à l'époque, voir Proulx, Entre France et Nouvelle-France, p. 85.

74 La pertinence de voyager en convoi repose sur la possibilité de « se défendre l'un l'autre, se placer sous la protection d'un bâtiment plus fort ». Proulx, Entre France et Nouvelle-France, p. 82 .

759 décembre 1715, Mémoire de Ruette d'Auteuil sur l'état présent du Canada, BAC-MG1C11A, bobine C2384, vol. 34, f 190v. Sur la carrière de cet acteur important en NouvelleFrance à l'époque, voir Marine Lelang, "Ruette d'Auteuil De Monceaux, FrançoisMadeleine-Fortuné », Dictionnaire biographique du Canada, vol. II (1701-1740).

76 Pour d'autres chiffres relatifs aux pertes et prises en temps de guerre, voir Proulx, Entre France et Nouvelle-France, pp. 79-80. 
le Machault. En deuxième lieu vient la Guerre de Succession d'Autriche avec trente-sept pertes (vingt-deux pourcent) et seulement douze durant la Guerre de Succession d'Espagne dont trois en 1711. En ce qui a trait aux ports d'attache, Bordeaux prédomine avec cinquante-quatre mentions (quarante pourcent), suivi de loin par La Rochelle avec vingt-et-une (quinze pourcent), Saint-Malo (19), le Havre (5) et Dunkerque (5). En Nouvelle-France, Québec est en tête avec onze, suivi par Louisbourg (5) et la Martinique (2).

Le tonnage de ces navires est mentionné à 136 occasions pour un total de 29523 (lorsqu'il y avait deux chiffres de mentionnés, nous optons pour le plus petit) pour une moyenne de 217 tonneaux par navire. Les plus gros sont deux navires du roi soit le Lys (1300 tonneaux) et le Belliqueux (1000 tonneaux). Le premier est pris le 8 juin 1755 et amené de Halifax en Angleterre, alors que le deuxième est capturé le 2 novembre 1758 au large de Bristol. L'on est également à même d'identifier seize ports de l'Atlantique Anglais où sont amarinées des prises françaises. Quatre d'entre eux prédominent et se trouvent en Angleterre soit Plymouth (16), Bristol (8), Falmouth (7) et Portsmouth (6). En Amérique du Nord, 5 prises sont conduites à St-John's (4) et Plaisance (1), devenue possession anglaise en 1713.

\section{Conclusion}

Cette recherche visait donc à aborder la problématique de la navigation transatlantique du $18^{\mathrm{e}}$ siècle dans l'Atlantique Français, plus spécifiquement le trajet France-Nouvelle-France. Dans un premier temps, après s'être familiarisés avec les impondérables et les étapes inquiétantes menant à des naufrages, nous avons aussi constaté l'impact qu'ils pouvaient avoir sur les maigres ressources des administrations coloniales, obligées d'intervenir en faveur des équipages et de la sauvegarde de la cargaison. Les observations de Pritchard, de Janzen, de Banks et des administrateurs coloniaux de l'île Royale, confirment qu'il y a sans doute des lacunes dans les connaissances hydrographiques et la formation technique des pilotes et des capitaines.

Dans un deuxième temps, l'utilisation du site web Vianney Campeau a permis de chiffrer sommairement ces pertes. Il s'agit cependant là d'un débat à finir puisqu'il demeure difficile de dire jusqu'à quel point l'historiographie a véritablement exploitée les sources avec cet objectif en tête. Finalement, à compter des années 1770, il semble que les autorités royales tentent de donner davantage de dents à la réglementation visant à améliorer la sécurité dans la pêche à Terre-Neuve. Quoiqu'on en dise, il semble poindre une esquisse de compassion envers les équipages bien que l'on ne peut évacuer la priorité de la France d'alors soit de maintenir des pêcheries à titre d'école de formation de matelots pour la Marine royale.

\section{Annexe I}

\section{L'ère des grandes réglementations des pêches 1770-1790}

Certes, l'ère post-Traité de Paris (1763) compte lui-aussi son lot de naufrages mais cette période se signale particulièrement par une volonté renouvelée de réglementer davantage la navigation et les activités de pêche française dans le Golfe du Saint-Laurent, 
surtout autour de Saint-Pierre et Miquelon et de Terre-Neuve. Il nous apparaît que ces derniers ont moins à faire avec la sécurité des équipages, qu'avec le contrôle des abus et détournements en cas de naufrages ou encore, pour préserver les intérêts des armateurs des ports de France. D'ailleurs Coindet est d'avis que l'Ordonnance de la Marine de 1681 , relativement aux naufrages, ne fait que très peu de place au « sort des équipages ». L'intérêt de "l'amirauté se concentre sur les marchandises et l'épave du navire, le recouvrement des effets sauvés constituant l'essentiel de la procédure ${ }^{77}$. » N'oublions pas le désir de la France de maintenir sa pépinière de matelots pour la Marine royale!

Il apparaît évident que le roi cherche d'abord à enrayer les abus des profiteurs tentant de s'enrichir à partir des biens rescapés des naufrages et pour ce faire, il questionne même l'intégrité de ses propres fonctionnaires. Ainsi, en ce même été 1769, un arrêt du Conseil d'État du roi défend à tous officiers d'amirauté d'ordonner ou d'accepter le dépôt d'effets et de marchandises provenant des «bris, naufrages et échouements dans les magasins appartenant à aucuns desdits officiers, ou dépendants des maisons qu'ils habitent ${ }^{78}$. » Dès l'année suivante, en 1770, le roi en rajoute dans l'espoir de mieux contrôler la gestion des naufrages et échouements. Ces nouvelles démarches constituent en fait des modifications à l'Ordonnance de la Marine de 1681, visant maintenant à « faire cesser les abus et rendre uniforme la manière de procéder en cette matière $^{79}$. »

De ces réflexions résulte éventuellement l'important règlement de 1773, qui « fixe la police des équipages des vaisseaux armés pour la pêche de la morue sur le Grand Banc et sur la côte de l'île de Terre-Neuve, dans les différentes circonstances où ils peuvent faire naufrage ${ }^{80}$. » D'emblée, cette ordonnance vise d'abord à fidéliser les équipages envers leur capitaine en cas de naufrage. On y défend clairement à l'équipage d'un navire naufragé d'abandonner son capitaine, ou encore de refuser de participer au sauvetage des débris et autres effets. En fait, ce règlement se déploie en deux sections soit la première concernant les naufrages survenant sur une côte autre que celle de TerreNeuve et la deuxième, pour les naufrages survenant « dans les glaces » ou banquises le long des côtes de cette île.

En principe, le règlement énonce les responsabilités réciproques de l'équipage, de l'armateur et du capitaine, en plus du sort réservé aux biens du navire. Des poursuites et amendes sont prévues contre ceux enfreignant le règlement, mais il demeure néanmoins que l'on se préoccupe de «l'intérêt du commerce » avant tout. Il faut d'abord demeurer à bord aussi longtemps que possible, à moins que le navire soit en " danger évident de perdition ». Une fois à terre, l'équipage doit obéir au capitaine au même titre que sur le navire, sous peine de «punition corporelle». N'empêche que le capitaine a le devoir de fournir des vivres à l'équipage ou de « payer des journées » de travail avec les

77 Sylvain Coindet, « Les naufrages sur l'île de Sein », p. 98.

7818 juin 1769, Arrêts, édits et ordonnances de la Marine, BAC-Série 3S2, vol. 107, dossier 296, 3 pages, Fonds du Service de la Marine à Vincennes.

7910 janvier 1770, Lettres patentes du roi, BAC-MG6-A17, vol. 13, f 20v-40, Versailles.

80 1773, Règlement qui fixe la police des équipages des vaisseaux armés pour la pêche à la morue, BAC-MG1-C11F, bobine F523, vol. 5, f 150-155v. 
« choses sauvées » du naufrage.

Si le naufrage survient lors du départ de France et que la saison n'est pas trop avancée, l'armateur peut transférer ce même équipage sur un ou d'autres navires et aux mêmes conditions. L'armateur doit aussi remplacer à ses frais ce que les matelots auraient perdu dans le naufrage. Par contre, ces mêmes matelots peuvent retrouver leur liberté s'ils remboursent les avances reçues de l'armateur. Si jamais un nouvel armement n'a pas lieu, l'armateur doit dédommager les matelots en leur versant une « équivalence au tiers des profits qu'il aurait pu faire ». Si la saison est trop avancée pour permettre un nouvel embarquement, l'armateur doit libérer les matelots de toute obligation et ils peuvent conserver les avances reçues. Aussi, ils n'ont pas à se compromettre ou s'engager auprès de cet armateur pour la saison suivante. Les matelots malades ou blessés suite au naufrage, n'ont pas à s'embarquer pour un deuxième armement la même saison.

Si le naufrage survient sur une côte éloignée du lieu d'armement ou lors du retour de la pêche, les matelots doivent être immédiatement « congédiés » et se rendre chez eux par leurs propres moyens. Mais c'est seulement s'il n'y a pas eu moyen de sauver des provisions pour leur subsistance. Dans des cas pareils, les commissaires de la Marine en France ou les Conseils de la Maison française à l'étranger tâcheront de leur fournir des provisions.

La seconde section du règlement s'attarde aux mesures à prendre lorsqu'un naufrage survient à Terre-Neuve, en raison des glaces tardives du printemps. Les capitaines doivent alors déployer tous les efforts possibles pour secourir l'équipage et les faire monter sur d'autres navires retournant en France. Les capitaines de ces vaisseaux ne peuvent refuser pareille requête. Il est également permis de répartir les matelots naufragés parmi d'autres équipages, sous la supervision de l'amiral du havre le plus près.

Mais si le naufrage se produit sur la côte même de Terre-Neuve, l'équipage demeure sous les ordres de son capitaine pour travailler à « sauver les débris », les effets de la cargaison, et faire la pêche si possible. Dans le cas contraire, l'amiral du havre accueillera les naufragés pour les répartir parmi les autres navires du havre ou des autres circonvoisins, à une distance maximale de dix lieues. Cette répartition se fait en proportion du nombre de chaloupes rattachées à chaque navire. Les officiers naufragés, pour leur part, sont également répartis mais seulement « en qualité de trancheurs ». Tout matelot ou officiers naufragés refusant de respecter les ordres de l'amiral du havre, devient passible d'une peine de trois mois de prison. Les capitaines à qui l'amiral demande d'accueillir des naufragés pour les intégrer à leurs équipages doivent obtempérer, sous peine d'une amende de 500 livres. Ces capitaines doivent aussi rembourser aux amiraux les vivres fournis aux naufragés durant le temps qu'ils demeurent sous leur responsabilité. Les capitaines recevant des naufragés doivent aussi accepter des novices et des mousses à proportion. Finalement, les naufragés ayant travaillé à la pêche des vaisseaux les ayant recueillis sont récompensés par un « lot à la mode du nord ${ }^{81}$.»

81 Ces règlements semblent fortement inspirés des recommandations d'un mémoire soumis par 
Après le règlement de 1773, il est intéressant d'examiner le contenu d'un document anonyme de 1785. Contrairement au contenu du règlement de 1773 , ce mémoire nous informe beaucoup plus sur les dangers auxquels s'exposent les navires de pêche arrivant trop hâtivement dans la saison à Terre-Neuve. Bien qu'anonyme, le mémoire en question semble provenir d'un capitaine expérimenté dans les dangers de la pêche terre-neuvienne. Surtout au moment de la dérive des banquises au printemps. Sa suggestion parapluie consiste à ce que «l'État règlemente les dates de départ des navires ", pour éviter les arrivées trop hâtives et dans des conditions mettant la vie des équipages en danger. Il est d'avis que les « malheurs » arrivés au printemps 1785 sur la « banquise puis sur la côte du Petit Nord», se comparent à ceux des campagnes de 1749 et 1750 . Les motifs des armateurs demeurent les mêmes; " l'avidité " voulant que les premiers arrivés puissent s'approprier les meilleurs havres où la pêche est réputée meilleure, avec les meilleures graves pour le séchage. Il pointe surtout du doigt les armateurs de Saint-Malo, qui tentent d'avoir tous leurs navires dans «le même havre sous un même chef ». Ils veulent ainsi être les premiers à ramener de la morue à Marseille pour toucher la prime. L'auteur du mémoire estime qu'il est inutile de faire partir les navires à la fin mars puisque la morue, elle, n'arrive que vers le 15 mai et parfois plus tard. Les équipages ont ainsi le temps de consommer un mois de vivres dans l'attente. En 1754, le gouvernement avait tenté de défendre le départ des navires de Granville, Saint-Malo et Saint-Brieuc avant le 25 avril, mais sans succès apparent ${ }^{82}$.

La section décrivant le processus de navigation à l'intérieur des banquises s'avère comme l'un des passages les plus intéressants de ce document. Notre auteur anonyme décrit deux scénarios troublants, se produisant souvent sur la côte de TerreNeuve au printemps ${ }^{83}$. D'abord, la situation où des capitaines vont «parcourir la banquise pour trouver un passage »; manœuvre dangereuse pouvant durer quelques jours. Si le capitaine aperçoit une " clairière dans la banquise ", il prend souvent la chance d'y entrer. Ce capitaine relate son expérience de 1753, alors qu'après avoir « côtoyé la banquise pendant quinze jours, j'entrouvrais un passage par lequel j'y passai 10 lieues en deux jours avec quarante autres navires, après quoi nous nous trouvâmes enfermés dans un lac d'une lieue de circonférence bordé de grosses glaces sur

la Chambre de commerce de Saint-Malo la même année. N.d. 1773, Mémoire sur le règlement proposé par la Chambre de commerce de Saint-Malo, BAC-MG1-C11F, bobine F523, vol. 5, f 18-20. D'autres réactions à ces nouvelles réglementations sont disponibles dans un document de mars 1774. Saint-Pierre et Miquelon, Analyse des réactions des armateurs et capitaines de navires aux nouvelles réglementations, BAC-MG1-C12, bobine F569, vol. 4, f 16-16v. Voir aussi un document du 18 décembre 1774, Rapport des réactions au règlement pour les cas de naufrage, BAC-MG1-C12, bobine F569, vol. 4, f 17-18v.

8228 décembre 1785, Correspondance générale, Terre-Neuve et les pêcheries, BAC-MG1C11F, bobine 523, vol. 5, f 109 .

83 Pour en connaître davantage sur la vie des capitaines de pêche français à Terre-Neuve à la fin du $18^{\mathrm{e}}$ siècle, voir le cas d'Eustache Le Pelley de Granville. Ses mémoires furent éditées par Monique Le Pelley Fonteney et Gilles Désiré dit Gosset sous le titre Mémoires d'un TerreNeuvas : Eustache Le Pelley Fonteney (1745-1820), Cully, France: OREP Éditions, 2011. 
lesquelles la mer faisait un bruit affreux. Pour comble de malheur, le brouillard survint et dura cinq jours ${ }^{84}$. » La situation est toute aussi désespérée pour des équipages de quinze à vingt hommes placés dans des bateaux par les navires. Ces embarcations sont parfois débarquées "dans les glaces à trente ou quarante lieues de terre ». Les plus malchanceux partent parfois à la dérive sur le Grand banc et ils en reviennent souvent « tous gelés » et aveugles ${ }^{85}$.

La question qui demeure est de savoir si ces règlements ont un effet bienveillant sur la sécurité des pêcheurs. En réalité, le nombre de pertes de vie est plutôt modeste durant le dix-huitième siècle, du moins si l'on s'en remet aux chiffres de Jean-François Brière. Ainsi, de 1725 à 1792, seulement 2,2\% des navires de pêche de Saint-Malo sont naufragés soit quatre-vingt onze sur 4.048. Pour Granville, de 1732 à 1792, on parle de $1,5 \%$ seulement soit cinquante quatre navires sur 3.567. En réalité, la majorité de ces accidents se produisent en Europe. Par exemple, dans l'industrie morutière Basque, quatre-vingt-dix pourcent des naufrages survenant entre 1689 et 1759 arrivent dans les eaux de ports européens. ${ }^{86}$

8428 décembre 1785, Correspondance générale, Terre-Neuve et les pêcheries, f 109.

85 Ibid., f $110 \mathrm{v}$.

86 Jean-François Brière, "The Safety of Navigation in the 18th Century French Cod Fisheries », Acadiensis, vol. 16, n 2 , 1987, pp. 85-86. 